\title{
Vaccine Hesitancy During the COVID-19 Pandemic: A Latent Class Analysis of Middle-Aged and Older US Adults
}

\author{
Abram L. Wagner ${ }^{1}$ (D) Julia M. Porth ${ }^{1} \cdot$ Zhenke Wu$^{2} \cdot$ Matthew L. Boulton $^{1} \cdot$ Jessica M. Finlay ${ }^{3,4}$. \\ Lindsay C. Kobayashi ${ }^{3}$
}

Accepted: 10 January 2022 / Published online: 25 January 2022

(c) The Author(s), under exclusive licence to Springer Science+Business Media, LLC, part of Springer Nature 2022

\begin{abstract}
It is important to distinguish between apprehensions that lead to vaccine rejection and those that do not. In this study, we (1) identifed latent classes of individuals by vaccination attitudes, and (2) compared classes of individuals by sociodemographic characteristics COVID-19 vaccination, and risk reduction behaviors. The COVID-19 Coping Study is a longitudinal cohort of US adults aged $\geq 55$ years $(n=2358)$. We categorized individuals into three classes based on the adult Vaccine Hesitancy Scale using latent class analysis (LCA). The associations between class membership and sociodemographic characteristics, COVID-19 vaccination, and other behaviors were assessed using chi-square tests. In total, $88.9 \%$ were Vaccine Acceptors, 8.6\% were Vaccine Ambivalent, and 2.5\% Vaccine Rejectors. At the end, 90.7\% of Acceptors, $62.4 \%$ of the Ambivalent, and $30.7 \%$ of the Rejectors had been vaccinated. The Ambivalent were more likely to be Black or Hispanic, and adopted social distancing and mask wearing behaviors intermediate to that of the Acceptors and Rejectors. Targeting the Vaccine Ambivalent may be an efficient way of increasing vaccination coverage. Controlling the spread of disease during a pandemic requires tailoring vaccine messaging to their concerns, e.g., through working with trusted community leaders, while promoting other risk reduction behaviors.
\end{abstract}

Keywords COVID-19 · Vaccination $\cdot$ Older people

\section{Introduction}

The World Health Organization (WHO) Strategic Advisory Group of Experts on Immunization (SAGE) defines vaccine hesitancy as "the reluctance or refusal to vaccinate

Jessica M. Finlay and Lindsay C. Kobayashi have contributed equally to this work.

Abram L. Wagner

awag@umich.edu

1 Department of Epidemiology, School of Public Health, University of Michigan, 1415 Washington Heights, Ann Arbor, MI 48109, USA

2 Department of Biostatistics, School of Public Health, University of Michigan, Ann Arbor, MI 48109, USA

3 Center for Social Epidemiology and Population Health, Department of Epidemiology, School of Public Health, University of Michigan, Ann Arbor, MI 48109, USA

4 Survey Research Center, Institute for Social Research, Social Environment and Health, University of Michigan, Ann Arbor, MI 48109, USA despite the availability of vaccines" [1, 2]. The SAGE working group acknowledges that vaccine hesitancy represents a spectrum of behaviors [1,2]. There is a large body of research describing specific vaccine concerns, including safety [3], scheduling [4, 5], and beliefs about natural versus vaccine-derived immunity [6].

By identifying vaccine hesitancy as one of ten threats to global health in 2019 [7], the WHO foreshadowed current challenges in COVID-19 vaccination. Within the US, vaccine supply has surpassed demand since around May 2021 [8, 9]. As of mid-October 2021 , only about $57 \%$ of the population (and $66 \%$ of those $\geq 12$ years old) have been fully vaccinated [10].

The current COVID-19 pandemic has unleashed an "infodemic" related to COVID-19 vaccine development and vaccine safety $[11,12]$. At the same time, concerns about new pharmaceutical products like COVID-19 vaccines are normal and expected, and Black and Hispanic individuals' attitudes towards pharmaceuticals may be shaped by experiences with medical discrimination [13]. Under the paradigm of shared decision-making, individuals should feel 
empowered to express their concerns about vaccines with health care providers $[14,15]$. Moreover, individuals could theoretically mitigate their risk through other risk-reduction behaviors, like social distancing [16] and mask wearing [17].

There is a need for more research on what concerns individuals have about vaccines among both those who accept and those who refuse vaccination. Within a longitudinal study of middle-aged and older adults in the US, we assessed vaccination attitudes at the start of 2021, and followed up for 4 months to determine when and if individuals were vaccinated against COVID-19. The aims of this study were to 1) identify latent classes of individuals based on their attitudes towards vaccination, and 2) compare classes of individuals by sociodemographic characteristics, COVID-19 vaccination, and other risk reduction behaviors. This research acknowledges that individuals could have a variety of concerns about vaccines, but it is necessary to separate out apprehensions that lead to rejecting vaccines versus issues with no such impact.

\section{Methods}

\section{Study Sample}

Data were from the COVID-19 Coping Study, which longitudinally followed adults aged $\geq 55$ years living in the United States on a monthly basis from April/May 2020 through April/May 2021 [18]. A non-probability, online recruitment strategy was used to identify and enroll participants through social media (Facebook, Instagram), organizational mailing lists, the NIH ResearchMatch database, and the University of Michigan Health Research database. At enrollment, participants completed a baseline online survey and were asked to complete follow-up online surveys each month for a year. Details on the study design and methodology are available elsewhere [18]. All study surveys are publicly available at: https://sph.umich.edu/covid19copingstudy/.

Participants were eligible for the present analysis if they responded to the questions regarding vaccine attitudes during the Jan/Feb 2021 wave and were followed up monthly for vaccination uptake until April/May 2021.

\section{Measures}

During the Jan/Feb 2021 wave, participants responded to the adult Vaccine Hesitancy Scale (aVHS), a list of ten statements about vaccines in general [19]. A list of the statements can be found in Fig. 1.

Vaccination status was collected each month beginning with the Jan/Feb 2021 wave. At this wave, participants were asked whether they had ever received a COVID-19 vaccine. For subsequent waves, they were asked whether they had received a new dose of vaccine in the past month.

We measured changes to risk reduction behaviors by comparing responses during the May/Jun 2020 and Dec 2020/ Jan 2021 waves. At both waves, participants were asked to report the number of days in the past week (0 days, 1-3, $4-6,7)$ they engaged in specific risk reduction behaviors: self-isolating, washing hands or using hand sanitizer more than normal, wearing a face mask, engaging in in-person face-to-face contact for 15 or more minutes. Constructed

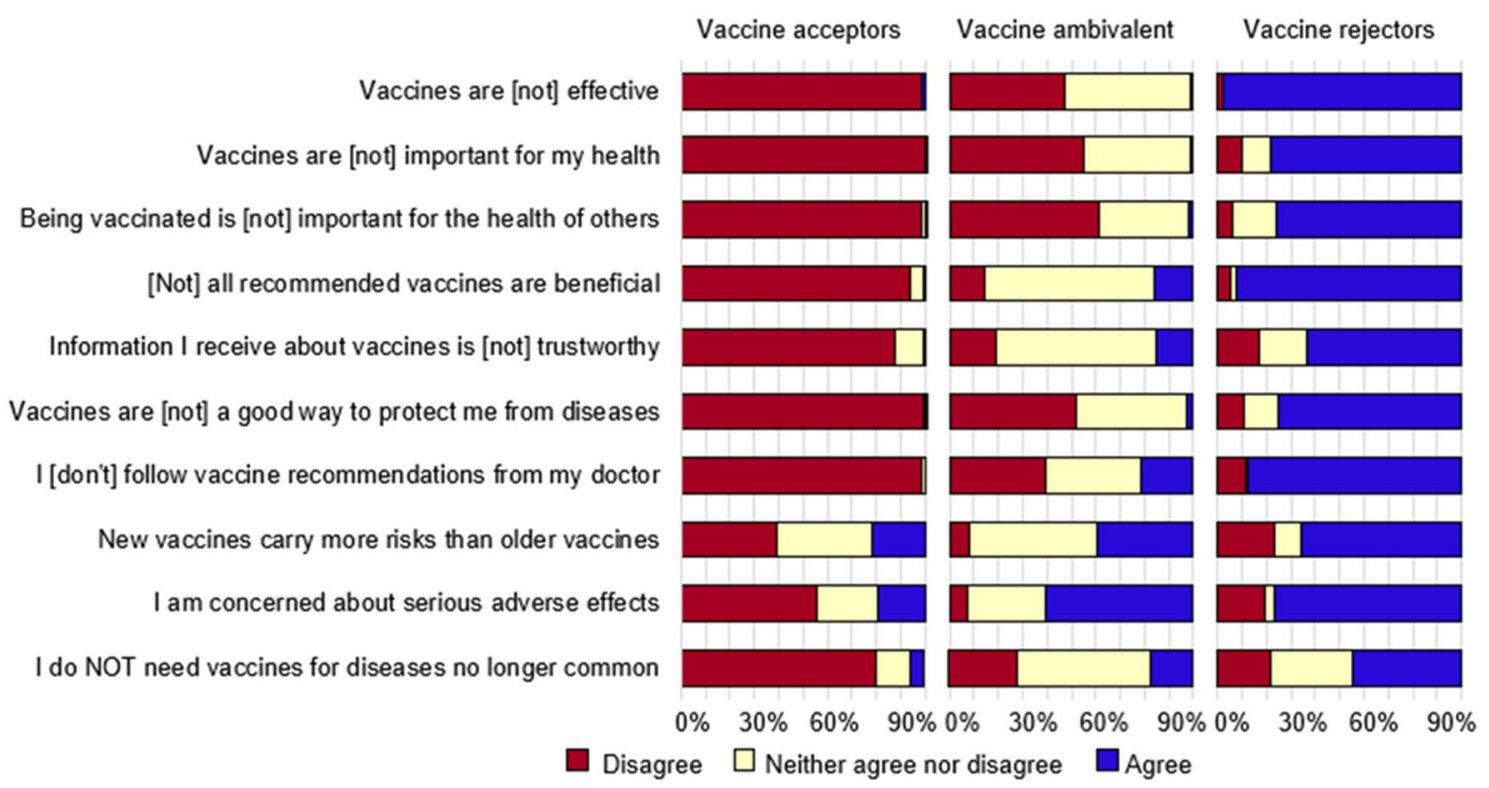

Fig. 1 Attitudes about vaccines stratified by latent class 
compliance variables comprised four categories: consistent complier (engaged in the activity 4-7 days in the past week at both the May/Jun 2020 and Dec 2020/Jan 2021 waves, consistent non-complier (engaged in the activity 0-3 days in the past week at both waves), rejuvenator (increased frequency of the activity from 0-3 days to 4-7 days between the waves), and fatiguer (decreased frequency of the activity from 4-7 days to 0-3 days between the waves).

Demographic covariates were assessed at the baseline (April/May 2020), and included sex (male, female), age ( $<65$ years old, $\geq 65$ years old), race (Black, white, other), ethnicity (Hispanic, not), education (some high school or high school diploma, some college or two-year associate degree, four-year college or university degree, postgraduate or professional degree), pre-COVID-19 employment status (employed, not employed, retired), relationship status (married or in a relationship, single), self-reported health (poor, fair, good, very good, excellent), and multi-morbidity (fewer than two chronic conditions, two or more chronic conditions).

\section{Statistical Analysis}

All analyses were weighted to account for sampling and participant attrition [18]. The final weights used in this analysis were the product of the sampling weight and the 9-month (Jan/Feb 2021 wave) attrition weight, as the vaccine hesitancy questions were asked at that wave.

Latent class analysis (LCA) was used to identify underlying groups of participants using their responses to the vaccine hesitancy questions. We ran three sets of LCA models with five-, three-, and two-level categorization of the survey questions (Supplemental Table 1). The first set of models operationalized the vaccine hesitancy statements as fivelevel variables (Strongly agree, Agree, Neither agree nor disagree, Disagree, Strongly Disagree). We ran four models within this set:

(1) Only vaccine hesitancy variables were included, treated as categorical variables.

(2) Vaccine hesitancy and other vaccine-related variables were included ("How often do you get vaccinated during the flu season?" And, "In deciding whether to get the COVID-19 vaccine, how important is ____?:" ( with responses: Other people in your community getting vaccinated, its being available for free, convenience in where you can get it, advice of your healthcare provider, advice of people you trust, your confidence in vaccine's safety, and your confidence in vaccine's effectiveness). All variables were treated categorically.

(3) Only vaccine hesitancy variables were included, treated as ordinal variables.
(4) Vaccine hesitancy variables and other vaccine-related questions were included, and treated as ordinal variables.

The second set of LCA models operationalized the vaccine hesitancy variables as three-level variables (Agree, Neither agree nor disagree, Disagree). The above four models were run in this set. In the final set of LCA models, the vaccine hesitancy variables were dichotomized into agree (Strongly agree, Agree) or do not agree (Neither agree nor disagree, Disagree, Strongly disagree). For all models, an increasing number of latent classes was allowed until the model failed to converge. AIC and BIC were used to assess model fit.

Within the optimal LCA model, participants were assigned to the latent class for which they had the highest posterior probability. Then, distributions of vaccination status, COVID-19-relevant behaviors, and sociodemographic characteristics across latent classes were compared. RaoScott chi-square tests of independence, which allow survey weights, were used to investigate associations between latent class assignment and the above factors. Holm-Bonferroni corrections were made to $\mathrm{p}$-values to account for multiple testing. Figures were created to show participants' vaccine attitudes among the entire eligible sample and within each latent class. Stata version 17.0 (College Station, TX, USA) was used for all analyses. The alpha level was 0.05 for significance.

\section{Results}

The original baseline sample included 4,401 individuals. Participants were excluded for the following reasons: did not respond to the Jan/Feb 2021 follow-up survey $(n=1954)$ or did not respond to all aVHS items $(n=89)$. The final analytic sample contained 2,358 participants. The demographic distribution of respondents is shown in Table 1.

For the LCA, we chose the three-level model with three latent classes as the optimal model for interpretability and which had relatively low AIC/BIC compared to most other models. Model fit statistics are shown in Supplemental Table 1.

Overall, most participants expressed pro-vaccine attitudes, agreeing that vaccines are effective, beneficial, and important to their own health and that of others in their community (Fig. 1). Upon examination of the vaccine opinions by latent class, we determined that one class held largely pro-vaccine attitudes (Vaccine Acceptors, $88.9 \%$ of participants), one class held more varied attitudes (Vaccine Ambivalent, $8.6 \%$ of participants), and one class held largely negative attitudes about vaccines (Vaccine Rejectors, 2.5\% of participants) (Fig. 1). Proportions of vaccine attitudes 
Table 1 Distributions of demographic characteristics in entire sample and within each Latent Class $^{\mathrm{a}}$

\begin{tabular}{|c|c|c|c|c|c|}
\hline Sociodemographic factor & $\begin{array}{l}\text { Total } \\
\text { sample } \\
(\mathrm{N}=2358)\end{array}$ & $\begin{array}{l}\text { Vaccine } \\
\text { Accep- } \\
\text { tors }\end{array}$ & $\begin{array}{l}\text { Vaccine } \\
\text { Ambiva- } \\
\text { lent }\end{array}$ & Vaccine Rejectors & $\begin{array}{l}\text { Adjusted } \\
\text { p-value }\end{array}$ \\
\hline Latent class membership & - & $88.9 \%$ & $8.6 \%$ & $2.5 \%$ & - \\
\hline Sex & & & & & 1 \\
\hline Male & $40.4 \%$ & $41.6 \%$ & $35.1 \%$ & $29.3 \%$ & \\
\hline Female & $59.6 \%$ & $58.4 \%$ & $64.9 \%$ & $70.7 \%$ & \\
\hline Age & & & & & 0.030 \\
\hline$<65$ years old & $38.4 \%$ & $36.0 \%$ & $51.5 \%$ & $54.4 \%$ & \\
\hline$\geq 65$ years old & $61.6 \%$ & $64.0 \%$ & $48.5 \%$ & $45.6 \%$ & \\
\hline Race & & & & & $<0.008$ \\
\hline Black & $5.9 \%$ & $5.2 \%$ & $12.1 \%$ & $2.6 \%$ & \\
\hline White & $87.2 \%$ & $89.3 \%$ & $68.9 \%$ & $94.7 \%$ & \\
\hline Other(s) & $6.9 \%$ & $5.5 \%$ & $19.0 \%$ & $2.7 \%$ & \\
\hline Ethnicity & & & & & 0.300 \\
\hline Hispanic or Latin(x) & $4.4 \%$ & $3.4 \%$ & $9.8 \%$ & $10.3 \%$ & \\
\hline Not Hispanic or Latin(x) & $95.6 \%$ & $96.6 \%$ & $90.2 \%$ & $89.7 \%$ & \\
\hline Education & & & & & 0.008 \\
\hline$\leq$ High school & $12.8 \%$ & $11.2 \%$ & $23.3 \%$ & $20.3 \%$ & \\
\hline Some college or associate's degree & $26.2 \%$ & $24.6 \%$ & $34.3 \%$ & $37.7 \%$ & \\
\hline Bachelor's degree & $28.3 \%$ & $28.5 \%$ & $27.0 \%$ & $27.3 \%$ & \\
\hline Graduate degree & $32.7 \%$ & $35.7 \%$ & $15.4 \%$ & $14.7 \%$ & \\
\hline Pre-COVID-19 employment status & & & & & $<0.008$ \\
\hline Employed & $36.6 \%$ & $34.2 \%$ & $46.3 \%$ & $64.2 \%$ & \\
\hline Not Employed & $10.2 \%$ & $8.7 \%$ & $20.8 \%$ & $11.9 \%$ & \\
\hline Retired & $53.2 \%$ & $57.1 \%$ & $32.9 \%$ & $23.9 \%$ & \\
\hline Relationship Status & & & & & 0.020 \\
\hline Married or in a relationship & $66.4 \%$ & $68.8 \%$ & $50.7 \%$ & $57.0 \%$ & \\
\hline Not married or in a relationship & $33.6 \%$ & $31.2 \%$ & $49.3 \%$ & $43.0 \%$ & \\
\hline Self-reported health & & & & & 1 \\
\hline Poor & $1.8 \%$ & $1.6 \%$ & $3.6 \%$ & $1.2 \%$ & \\
\hline Fair & $11.3 \%$ & $10.8 \%$ & $16.8 \%$ & $5.4 \%$ & \\
\hline Good & $31.3 \%$ & $31.5 \%$ & $29.2 \%$ & $33.5 \%$ & \\
\hline Very good & $37.4 \%$ & $37.8 \%$ & $34.9 \%$ & $36.4 \%$ & \\
\hline Excellent & $18.2 \%$ & $18.3 \%$ & $15.5 \%$ & $23.5 \%$ & \\
\hline Multi-morbidity & & & & & 1 \\
\hline Fewer than 2 chronic conditions & $85.7 \%$ & $85.0 \%$ & $87.9 \%$ & $94.1 \%$ & \\
\hline 2 or more chronic conditions & $14.3 \%$ & $15.0 \%$ & $12.1 \%$ & $5.9 \%$ & \\
\hline
\end{tabular}

${ }^{a}$ Weighted by 9 -month sample weight $\times$ attrition weight

${ }^{\mathrm{b}}$ Holm-Bonferroni-adjusted p-value of Rao-Scott Chi-square, which takes sampling and attrition weighting into account by latent class membership can be found in Supplemental Table 2. For Vaccine Acceptors, there was still substantial hesitancy about newer vaccines carrying more risks $(22.1 \%$ agreed), and concern of serious adverse effects (19.2\%). Among the Vaccine Ambivalent, there were substantive concerns about whether all vaccines were beneficial (only $14.2 \%$ agreed), and whether information about vaccines was trustworthy (19.3\% agreed). Compared to Vaccine Acceptors, the Vaccine Ambivalent expressed greater concerns about serious adverse effects and newer vaccines carrying more risks.
Across all ten items, Vaccine Rejectors expressed hesitancy to a great degree.

There were significant associations between latent class membership and vaccination status at each wave of followup (Table 2). During Jan/Feb 2021, 27.3\% of Acceptors, 9.1\% of the Ambivalent, and $12.2 \%$ of Rejectors were vaccinated. By Apr/May 2021, these numbers were $90.7 \%, 62.4 \%$, and $30.7 \%$, respectively.

The relationships between latent class membership for vaccination attitudes and risk reduction behavior varied 
Table 2 Vaccination status at 9-, 10-, 11-, and 12-month follow-up in entire sample and within each Latent Class ${ }^{\mathrm{a}}$

\begin{tabular}{|c|c|c|c|c|c|}
\hline & Total sample & Vaccine acceptors & $\begin{array}{l}\text { Vaccine } \\
\text { ambivalent }\end{array}$ & Vaccine rejectors & $\begin{array}{l}\text { Adjusted } \\
\text { p-value }^{b}\end{array}$ \\
\hline Vaccination status Jan/Feb 2021 & & & & & $<0.004$ \\
\hline Vaccinated & $24.7 \%$ & $27.3 \%$ & $9.1 \%$ & $12.2 \%$ & \\
\hline Not vaccinated & $75.3 \%$ & $72.7 \%$ & $90.9 \%$ & $87.8 \%$ & \\
\hline Vaccination status Feb/Mar 2021 & & & & & $<0.004$ \\
\hline Vaccinated & $50.2 \%$ & $55.1 \%$ & $22.9 \%$ & $18.9 \%$ & \\
\hline Not vaccinated & $49.8 \%$ & $44.9 \%$ & $77.1 \%$ & $81.1 \%$ & \\
\hline Vaccination status Mar/Apr 2021 & & & & & $<0.004$ \\
\hline Vaccinated & $73.0 \%$ & $78.3 \%$ & $47.3 \%$ & $28.1 \%$ & \\
\hline Not vaccinated & $27.0 \%$ & $21.7 \%$ & $52.7 \%$ & $71.9 \%$ & \\
\hline Vaccination status Apr/May 2021 & & & & & $<0.004$ \\
\hline Vaccinated & $85.3 \%$ & $90.7 \%$ & $62.4 \%$ & $30.7 \%$ & \\
\hline Not vaccinated & $14.7 \%$ & $9.3 \%$ & $37.6 \%$ & $69.3 \%$ & \\
\hline
\end{tabular}

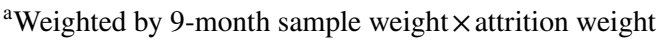

${ }^{\mathrm{b}}$ Holm-Bonferroni-adjusted p-value of Rao-Scott Chi-square, which takes sampling and attrition weighting into account

over time (Table 3 and Supplementary Table 3). There was a significant relationship by risk reduction behavior except for the measure of having face-to-face contact. In general, the Vaccine Ambivalent had behaviors intermediate to Acceptors and Rejectors at baseline in spring 2020, but that they also had a high degree of fatigue in these behaviors over time, trending towards Vaccine Rejectors by the end of 2020. For example, in May/Jun 2020, 46.6\% of the

Table 3 COVID-19 behaviors in entire sample and within each Latent Class ${ }^{\mathrm{a}}$

\begin{tabular}{|c|c|c|c|c|c|}
\hline & Total sample & Vaccine acceptors & $\begin{array}{l}\text { Vaccine } \\
\text { ambivalent }\end{array}$ & Vaccine rejectors & $\begin{array}{l}\text { Adjusted } \\
\text { p-value }^{b}\end{array}$ \\
\hline Days in the past week spent self-isolating & & & & & 0.008 \\
\hline Consistent complier & $64.9 \%$ & $67.7 \%$ & $51.6 \%$ & $32.1 \%$ & \\
\hline Consistent non-complier & $9.7 \%$ & $8.1 \%$ & $18.9 \%$ & $22.5 \%$ & \\
\hline Rejuvenator & $8.1 \%$ & $7.8 \%$ & $11.6 \%$ & $2.5 \%$ & \\
\hline Fatiguer & $17.3 \%$ & $16.4 \%$ & $17.9 \%$ & $42.9 \%$ & \\
\hline $\begin{array}{l}\text { Days in the past week spent washing hands or using hand } \\
\text { sanitizer more than normal }\end{array}$ & & & & & 0.027 \\
\hline Consistent complier & $72.2 \%$ & $74.4 \%$ & $62.0 \%$ & $40.1 \%$ & \\
\hline Consistent non-complier & $10.2 \%$ & $10.2 \%$ & $7.6 \%$ & $23.9 \%$ & \\
\hline Rejuvenator & $5.2 \%$ & $4.4 \%$ & $11.4 \%$ & $5.3 \%$ & \\
\hline Fatiguer & $12.4 \%$ & $11.0 \%$ & $19.0 \%$ & $30.7 \%$ & \\
\hline Days in the past week participant has worn a face mask & & & & & 0.040 \\
\hline Consistent complier & $72.2 \%$ & $74.4 \%$ & $62.0 \%$ & $40.1 \%$ & \\
\hline Consistent non-complier & $10.2 \%$ & $10.2 \%$ & $7.6 \%$ & $23.9 \%$ & \\
\hline Rejuvenator & $5.2 \%$ & $4.4 \%$ & $11.4 \%$ & $5.3 \%$ & \\
\hline Fatiguer & $12.4 \%$ & $11.0 \%$ & $19.0 \%$ & $30.7 \%$ & \\
\hline $\begin{array}{l}\text { Days in the past week with in-person face-to-face contact } \\
\text { for } 15+\text { minutes }\end{array}$ & & & & & 1 \\
\hline Consistent complier & $32.0 \%$ & $31.5 \%$ & $34.2 \%$ & $38.8 \%$ & \\
\hline Consistent non-complier & $23.4 \%$ & $23.3 \%$ & $23.3 \%$ & $27.5 \%$ & \\
\hline Rejuvenator & $4.6 \%$ & $4.7 \%$ & $4.0 \%$ & $3.1 \%$ & \\
\hline Fatiguer & $40.0 \%$ & $40.5 \%$ & $38.5 \%$ & $30.6 \%$ & \\
\hline
\end{tabular}

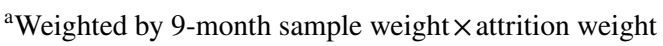

${ }^{\mathrm{b}}$ Holm-Bonferroni-adjusted p-value of Rao-Scott Chi-square, which takes sampling and attrition weighting into account 
Ambivalent self-isolated for 7 days (compared to $48.9 \%$ of Vaccine Rejectors and $64.5 \%$ of Vaccine Acceptors). By Dec 2020/Jan 2021, these proportions dropped among all groups (31.6\% of the Vaccine Ambivalent self-isolated for 7 days, compared to $20.3 \%$ of Vaccine Rejectors and $46.3 \%$ of Vaccine Acceptors, see Supplementary Table 3).

Among latent classes there were statistically significant differences in the distributions of age, race, education, pre-COVID-19 employment status, and relationship status (Table 1). Compared to Vaccine Acceptors, a higher proportion of Vaccine Ambivalent and Vaccine Rejectors were younger than 65 years old $(36.0 \%, 51.5 \%, 54.4 \%$, respectively). There were higher proportions of Black $(12.1 \%)$ and other race $(19.0 \%)$ participants among the Ambivalent compared to both Acceptors (5.2\% Black, 5.5\% other) and Rejectors (2.6\% Black, 2.7\% other). Among Acceptors, the highest proportion of participants had a postgraduate or professional degree (35.7\%), whereas some college or two-year associate degrees were the most common level of education among the Ambivalent (34.3\%) and Rejectors (37.7\%). Most Rejectors were employed before the pandemic $(64.2 \%)$ compared to less than half of the Ambivalent (46.3\%) and approximately one-third of Acceptors (34.2\%). Additionally, more Acceptors were in a relationship $(68.8 \%)$ than either the Ambivalent (50.7\%) or Rejectors (57.0\%).

\section{Discussion}

Low acceptance of a COVID-19 vaccine by segments of the population could foster continued outbreaks and amplify challenges to controlling the spread of SARS-CoV-2. In the US, where supply of COVID-19 vaccine currently exceeds demand, it is important to identify what vaccine-related beliefs are associated with actual vaccination. In a longitudinal study of middle-aged and older adults in the US, we found a large majority were Vaccine Acceptors (for vaccines in general) and had received a COVID-19 vaccine by April/ May 2021. The Vaccine Ambivalent will be important targets in the identification of strategies to increase population vaccine uptake, especially as COVID-19 vaccination booster programs roll out.

As measured through a latent class analysis of an adult Vaccine Hesitancy Scale [19], almost 9 in 10 adults fell into the Vaccine Acceptor class, and were among the first to receive a COVID-19 vaccine when it was introduced in the United States. The adult Vaccine Hesitancy Scale measures hesitancy about vaccines in general, not for COVID-19 vaccines, but a previous study also found high overlap between patterns of vaccine hesitancy in general and rejection for COVID-19 vaccine specifically [20]. Another study of adults in Tennessee found that many individuals believe they have not changed their attitudes towards vaccines because of the pandemic [21], which suggests that many vaccine beliefs are deeply entrenched.

We also want to highlight the sociodemographic differences across these classes. A previous survey found younger adults, women, non-Hispanic Black persons, adults not in cities, and adults with lower educational attainment, with lower income, and without health insurance were the most likely to report not wanting to receive a COVID-19 vaccine [22]. In our study, the Vaccine Ambivalent were more likely to be Black or Hispanic than the Vaccine Acceptors or Rejectors. Prior to the COVID-19 pandemic, adult influenza vaccination rates in the US were about 10 percentage points lower in Hispanic and Black Americans compared to their white counterparts [23], which could be due to vaccine hesitancy, but also issues of access, affordability [24], and racism experienced within the healthcare system [24]. To mitigate disparities in who contracts SARS-CoV-2, it will be important to increase vaccination uptake in Black and Hispanic Americans, for instance by involving trusted community leaders in delivering pro-vaccine messaging [25].

We found that a greater share of the middle-aged and older population was Vaccine Ambivalent than Vaccine Rejectors, indicating they would be a more responsive target for strategies to increase population vaccine uptake. Past research into pediatric vaccines has introduced the concept of "fence-sitters" [26], who are those parents who have concerns about vaccines and may delay or selectively choose certain vaccines based on their own research. A response to these individuals should target their particular concerns[27] and not assume that the individual has a knowledge deficit [26]. The present study adds several pieces of information about the Vaccine Ambivalent, to distinguish them from Acceptors or Rejectors. First, this study showed that unlike Rejectors and like Acceptors, the Vaccine Ambivalent believe that being vaccinated could be important for the health of others. Tailoring to the individual will be important here. According to the "protector" schema, whom the individual is protecting matters; vaccine promotional materials can highlight protection against a concrete person, like a close relative, instead of a generic "other" [28]. Second, we found that many in the Vaccine Ambivalent group do not trust doctors, and so these individuals may not always be the best delivery mechanism.

The Vaccine Ambivalent had other concerns, such as the riskiness of newer vaccines, that has been echoed in previous studies. In a review of vaccine hesitancy studies during the COVID-19 pandemic, Troiano found many studies revealed substantial concerns about the speed of vaccine development [29]. A survey of adults in December 2020 found that among those not intending to be vaccinated, the main reasons included concerns about side effects and safety $(29.8 \%)$ and that the vaccine was developed rapidly (10.4\%) [22]. In a study by Nguyen et al. [22], $14.5 \%$ of adults mentioned 
wanting to wait and see if the vaccine is safe and effective prior to receiving it. The slower uptake of COVID-19 vaccination among the Vaccine Ambivalent in our study points to this "wait-and-see" approach, and is in line with the importance of positive experiences (such as personally knowing individuals safely vaccinated) in vaccine decision-making [30].

Beyond vaccination, the Vaccine Acceptors also adopted more risk reduction behaviors, including washing hands, wearing masks, and social distancing. The Vaccine Ambivalent were more likely to engage in behaviors to reduce risk of viral spread than Vaccine Refusers, which could be a way to partially compensate for not receiving a vaccine by reducing risks through other behaviors. Yet, other papers have also shown a correlation between vaccination intent and social distancing and mask usage [31]. These findings may speak to a more general "COVID-19 social identity" [31]. in showing outward behaviors congruent with groups believing that the pandemic is real. Overall, more research is needed on how to encourage behaviors that can limit spread of disease among those who want to "wait and see" prior to obtaining a vaccine.

\section{Strengths and Limitations}

Non-probability sampling strategy means that our sample is not population-representative. Reassuringly, the proportion of vaccinated adults in our sample by the end of May 2021 is similar to that of the general US population of this age range at the same point in time ( $85 \%$ of our sample, compared to $88 \%$ of those aged $65-74$ and $84 \%$ of those aged $\geq 75$ in the general population[10]). The study sample was weighted to the general US population aged $\geq 55$, but the sample may not represent individuals who were too sick to participate, or who could not access the Internet. The population sampling weight decreases selection and other sampling biases, so while our results may not be representative of the general population, the estimated relationships should be minimally biased. Individuals may have responded in a way they felt was socially desirable for vaccine opinions, vaccination status, and risk reduction behaviors. A strength of the study was the large sample size and broad geographic scope, with representation from all 50 US states and the District of Columbia. Data collection began early in the pandemic and continued longitudinally, meaning we were able to capture changes in behaviors throughout course of the pandemic.

\section{Conclusions}

Most middle-aged and older Americans have positive attitudes about vaccines. Vaccine Ambivalent adults appear more similar to Vaccine Rejectors than Acceptors in terms of their demographic characteristics and in their initial vaccination behaviors. As the pandemic continued through 2020 and into 2021, the Vaccine Ambivalent maintained their engagement in risk reduction behaviors more than Rejectors. Although descriptive, these findings have important implications for public health messaging and planning. Notably, we observed that, over time, Vaccine Ambivalent middle-aged and older adults seemed to move towards the vaccination behaviors of Vaccine Acceptors. This finding suggests that, with appropriate messaging and engagement of trusted leaders (not necessarily physicians) in conversations about preventive measures, many, or at least some, Vaccine Ambivalent individuals may be convinced to get vaccinated and engage in other preventive behaviors.

Supplementary Information The online version contains supplementary material available at https://doi.org/10.1007/s10900-022-01064-w.

Author Contributions Conceptualization: ALW, JMF, LCK; Formal analysis and investigation: JMP, ZW; Writing - original draft preparation: ALW, JMP; Writing - review and editing: ZW, MLB, JMF, LCK; Funding acquisition: JMF, LCK.

Funding This project was supported by an award from the National Science Foundation, Division of Social and Economic Sciences (\#2027836), the National Institute on Aging (\#P30AG012846 and \#F32AG064815), and the Michigan Institute for Clinical and Health Research Postdoctoral Translational Scholar Program (\#UL1 TR002240-02).

Data availability Data are available at: https://doi.org/10.3886/E1310 $22 \mathrm{~V} 1$.

\section{Declarations}

Conflict of interest The authors declare that they have no conflict of interest.

Ethical Approval The University of Michigan Health Sciences and Behavioral Sciences Institutional Review Board (IRB \# HUM00179632) provided ethical approval to the COVID-19 Coping Study. In this internet-based survey, participants read an informed consent form and had to click "agree" before seeing the questionnaire.

\section{References}

1. The Strategic Advisory Group of Experts (SAGE). (2014). Report of the SAGE working group on vaccine hesitancy. Retrieved June 14, 2018, from http://www.who.int/immunization/sage/meetings/ 2014/october/SAGE_working_group_revised_report_vaccine_ hesitancy.pdf.

2. MacDonald, N. E., \& SAGE Working Group on Vaccine Hesitancy. (2015). Vaccine hesitancy: Definition, scope, and determinants. Vaccine, 33, 4161-4164.

3. Wagner, A. L., Huang, Z., Ren, J., Laffoon, M., Ji, M., Pinckney, L. C., \& Zikmund-Fisher, B. J. (2021). Vaccine hesitancy and concerns about vaccine safety and effectiveness in Shanghai China. American Journal of Preventive Medicine, 60(1), S77-S86. https://doi.org/10.1016/j.amepre.2020.09.003 
4. Wagner, A. L., Boulton, M. L., Sun, X., Huang, Z., Harmsen, I. A., Ren, J., \& Zikmund-Fisher, B. J. (2017). Parents' concerns about vaccine scheduling in Shanghai, China. Vaccine, 35(34), 4362-4367. https://doi.org/10.1016/j.vaccine.2017.06.077

5. Huang, Z., Wagner, A. L., Lin, M., Sun, X., Zikmund-Fisher, B. J., Boulton, M. L., \& Prosser, L. A. (2020). Preferences for vaccination program attributes among parents of young infants in Shanghai, China. Human Vaccines and Immunotherapeutics. https://doi.org/10.1080/21645515.2020.1712937

6. Sun, X., Huang, Z., Wagner, A. L. A. L., Prosser, L. A. L. A., Xu, E., Ren, J., \& Zikmund-Fisher, B. J. B. J. (2018). The role of severity perceptions and beliefs in natural infections in Shanghai parents' vaccine decision-making: A qualitative study. BMC Public Health, 18(1), 813. https://doi.org/10.1186/s12889-018-5734-9

7. World Health Organization. (2019). Ten threats to global health in 2019. 2019. Retrieved January 22, 2019, from https://www.who. int/emergencies/ten-threats-to-global-health-in-2019.

8. Rattner, N. (2021, April). Covid vaccinations are slowing in the U.S. as supply outstrips demand. How states are targeting who's left. $C N B C$. Retrieved October 14, 2021, from https://www.cnbc. com/2021/04/30/covid-vaccinations-in-us-are-slowing-as-supplyoutstrips-demand.html.

9. Kates, J., Levitt, L., \& Michaud, J. (2021). Supply vs Demand: When Will the Scales Tip on COVID-19 Vaccination in the U.S.? Kaiser Family Foundation. Retrieved October 14, 2021, from https://www.kff.org/policy-watch/supply-vs-demand-when-willthe-scales-tip-on-covid-19-vaccination-in-the-u-s/.

10. Centers for Disease Control and Prevention. (2021). COVID Data Tracker - Vaccinations. Retrieved October 14, 2021, from https:// covid.cdc.gov/covid-data-tracker/\#datatracker-home.

11. Islam, M. S., Sarkar, T., Khan, S. H., Mostofa Kamal, A.-H., Hasan, S. M. M., Kabir, A., \& Seale, H. (2020). COVID-19related infodemic and its impact on public health: A global social media analysis. The American Journal of Tropical Medicine and Hygiene, 103(4), 1621-1629. https://doi.org/10.4269/ajtmh. 20-0812

12. Biasio, L. R., Bonaccorsi, G., Lorini, C., \& Pecorelli, S. (2020). Assessing COVID-19 vaccine literacy: A preliminary online survey. Human Vaccines and Immunotherapeutics. https://doi.org/ 10.1080/21645515.2020.1829315

13. Evans, A., Webster, J., \& Flores, G. (2021). Partnering with the faith-based community to address disparities in COVID-19 vaccination rates and outcomes among US black and Latino populations. JAMA, 326(7), 609-610. https://doi.org/10.1001/JAMA. 2021.12652

14. Durand, M. A., Scalia, P., \& Elwyn, G. (2021). Can shared decision making address COVID-19 vaccine hesitancy? BMJ Evidence-Based Medicine. https://doi.org/10.1136/ bmjebm-2021-111695

15. Witteman, H. O. (2015). Addressing vaccine hesitancy with values. Pediatrics, 136(2), 215-217. https://doi.org/10.1542/peds. 2015-0949

16. Jarvis, C. I., Van Zandvoort, K., Gimma, A., Prem, K., Klepac, P., Rubin, G. J., \& Edmunds, W. J. (2020). Quantifying the impact of physical distance measures on the transmission of COVID-19 in the UK. BMC Medicine, 18(1), 124. https://doi.org/10.1186/ s12916-020-01597-8

17. Trogen, B., \& Caplan, A. (2021). Risk compensation and COVID19 vaccines. Annals of Internal Medicine, 174(6), 858-859. https://doi.org/10.7326/m20-8251

18. Kobayashi, L. C., Shea, B. Q. O., Kler, J. S., Nishimura, R., Palavicino-, C. B., Eastman, M. R., \& Finlay, J. M. (2021). Cohort profile: The COVID-19 Coping methods Study, a longitudinal mixed-methods - study of middle-aged and older adults ' mental health and well-being during the COVID-19 pandemic in the USA. British Medical Journal Open, 11, 1-11. https://doi.org/ 10.1136/bmjopen-2020-044965

19. Akel, K. B., Masters, N. B., Shih, S.-F., Lu, Y., \& Wagner, A. L. (2021). Modification of a vaccine hesitancy scale for use in adult vaccinations in the United States and China. Human Vaccines \& Immunotherapeutics, 17(8), 2639-2646. https://doi.org/10.1080/ 21645515.2021.1884476

20. Shih, S.-F., Wagner, A. L., Masters, N. B., Prosser, L. A., Lu, Y., \& Zikmund-Fisher, B. J. (2021). Vaccine hesitancy and rejection of a vaccine for the novel coronavirus in the United States. Frontiers in Immunology. https://doi.org/10.3389/fimmu.2021.558270

21. Gatwood, J., McKnight, M., Fiscus, M., Hohmeier, K. C., \& Chisholm-Burns, M. (2021). Factors influencing likelihood of COVID-19 vaccination: A survey of Tennessee adults. American Journal of Health-System Pharmacy, 78(10), 879-889. https://doi. org/10.1093/ajhp/zxab099

22. Nguyen, K. H., Srivastav, A., Razzaghi, H., Williams, W., \& Lindley, M. C. (2021). COVID-19 vaccination intent, perceptions, and reasons for not vaccinating among groups prioritized for early vaccination-United States, September and December 2020. MMWR. Morbidity and Mortality Weekly Report, 70, 217-222.

23. Grohskopf, L. A., Liburd, L. C., \& Redfield, R. R. (2020). Addressing influenza vaccination disparities during the COVID19 pandemic. JAMA, 324(11), 1029. https://doi.org/10.1001/jama. 2020.15845

24. Thomson, A., Robinson, K., \& Vallée-Tourangeau, G. (2016). The 5As: A practical taxonomy for the determinants of vaccine uptake. Vaccine, 34(8), 1018-1024. https://doi.org/10.1016/j.vacci ne.2015.11.065

25. Jamison, A. M., Quinn, S. C., \& Freimuth, V. S. (2019). "You don't trust a government vaccine": Narratives of institutional trust and influenza vaccination among African American and white adults. Social Science and Medicine, 221, 87-94. https://doi.org/ 10.1016/j.socscimed.2018.12.020

26. Leask, J. (2011). Target the fence-sitters. Nature, 473, 443-445.

27. Poland, C. M., \& Poland, G. A. (2011). Vaccine education spectrum disorder: The importance of incorporating psychological and cognitive models into vaccine education. Vaccine, 29(37), 6145-6148. https://doi.org/10.1016/j.vaccine.2011.07.131

28. Oganesyan, N. (2021). Positive COVID-19 Messaging on TV Can Persuade Resistant Viewers, The Protector Coalition Says. Retrieved February 1, 2021, from https://variety.com/2021/tv/ news/positive-tv- messaging-coronavirus-the-protector-coalition-1234885193/

29. Troiano, G., \& Nardi, A. (2021). Vaccine hesitancy in the era of COVID-19. Public Health. https://doi.org/10.1016/j.puhe.2021. 02.025

30. Wagner, A. L., Pinckney, L. C., \& Zikmund-Fisher, B. J. (2020). Vaccine Decision-making and Vaccine Hesitancy. In M. L. Boulton \& R. B. Wallace (Eds.), Maxcy-Rosenau-Last Public Health and Preventive Medicin2 (16th ed.). New York: McGraw-Hill Publishing.

31. Latkin, C. A., Dayton, L., Yi, G., Colon, B., \& Kong, X. (2021). Mask usage, social distancing, racial, and gender correlates of COVID-19 vaccine intentions among adults in the US. PLoS ONE, 16, 1-11. https://doi.org/10.1371/journal.pone.0246970

Publisher's Note Springer Nature remains neutral with regard to jurisdictional claims in published maps and institutional affiliations. 